\title{
Ghd8 controls rice photoperiod sensitivity by forming a complex that interacts with Ghd7
}

\author{
Peng Wang ${ }^{1,2+}$, Rong Gong ${ }^{1+}$, Ying Yang ${ }^{1,2}$ and Sibin Yu ${ }^{1 *}$ (D)
}

\begin{abstract}
Background: Flowering time is one of the most important agronomic characteristics that ultimately determine yield potential and eco-geographical adaptation in crops. Ghd8 and Ghd7, two major flowering genes, have similar functions and large pleiotropic effects in controlling the heading date, plant height and grain yield of rice. However, these gene interactions at the genetic and molecular levels have not been determined to date.

Results: In this study, we investigated the genetic interaction between Ghd8 and Ghd7 by using a set of near-isogenic lines and a panel of natural germplasm accessions in rice. We found that Ghd8 affected multiple agronomic traits in a functional Ghd7-dependent manner. Both functional Ghd8 and Ghd7 are pivotal for rice photoperiod sensitivity controlled by Hd1 and Hd3a. GHD8 could form a heterotrimeric complex with HD1 and OsHAP5b to activate the transcription of Ghd7 by binding directly to the promoter region of Ghd7, which contains the CCAAT-box motif.

Conclusions: The results of this study help to elucidate the genetic and molecular bases of Ghd8 and Ghd7 interactions, indicating that Ghd8 acts upstream of Ghd7 to activate its transcription, which inhibits Hd3a expression and thus affects flowering time and rice adaptation.
\end{abstract}

Keywords: Photoperiod sensitivity, Flowering time, Molecular interaction, CCAAT-box motif, Rice

\section{Background}

Photoperiod sensitivity (PS) is defined as the developmental responses of plants to the relative lengths of light and dark periods and confers on many plant species the ability to adapt to a range of growing season periods by means of adjusting flowering time. Flowering time or heading date (HD) is one of the most important agronomic traits in crops. The probable initiation of flowering time in response to climate change largely determines the crop yield potential [1]. This property is observed because a positive correlation was found between grain yield and flowering time in various natural conditions [2], and either too early or too late flowering might cause reduced grain yield. It has been reported that the photoperiod insensitivity in crops due to lossof-function mutants or weak alleles of flowering time

\footnotetext{
* Correspondence: ysb@mail.hzau.edu.cn

†Peng Wang and Rong Gong contributed equally to this work.

'National Key Laboratory of Crop Genetic Improvement, College of Plant Science and Technology, Huazhong Agricultural University, Wuhan, China Full list of author information is available at the end of the article
}

genes restricts their distributions at specific environmental conditions, such as high latitudes [3-6].

The regulatory mechanisms of flowering time have been extensively studied in Arabidopsis and rice [7, 8]. As a short-day plant, rice (Oryza sativa L.) can flower promptly under short-day (SD) conditions and flower relatively late under long-day (LD) conditions. Two independent gene pathways have been reported to be involved in regulating flowering time under both conditions. The OsGI-Hd1-Hd3a (rice GIGANTEA, Heading date 1 and Heading date 3a) signaling pathway in rice is evolutionarily conserved as the GI-CO-FT (GIGANTEA, CONSTANS, and FLOWERING LOCUS T) pathway in Arabidopsis. Among these genes, the expression of florigen genes in the downstream pathway, such as $H d 3 a / F T$ plays central roles in determining flowering time. High expression of $H d 3 a / F T$ strongly accelerates flower time, and downregulation of its expression delays or prevents flowering $[9,10]$. In recent years, several novel flowering genes have been identified in rice; they have no orthologs in the Arabidopsis genome

(c) The Author(s). 2019 Open Access This article is distributed under the terms of the Creative Commons Attribution 4.0 International License (http://creativecommons.org/licenses/by/4.0/), which permits unrestricted use, distribution, and 
and constitute a rice-specific flowering pathway. For example, Ghd7 (Grain number, plant height and heading date 7), a homolog group of CO, CO-like, and TOC1 (CCT)-domain proteins, was identified as a repressor of flowering through inhibiting $H d 3 a$ under LD conditions [11]. Ehd1 (Early heading date 1) was identified as the rice-specific flowering signal integrator and acts upstream of $\mathrm{Hd} 3 \mathrm{a}$ [12]. In addition, several flowering time genes have recently been identified to participate in either of the two main independent signaling pathways or even link them. Ghd7.1/OsPRR37 harboring a conserved CCT domain was reported to inhibit Ehd 1 and $H d 3 a$ only under LD conditions but was independent of $H d 1$. Ghd8/DTH8 (Grain number, plant height and heading date 8), encoding a CCAAT-box binding factor, known as a HAP3/NF-YB protein, was identified as a major effect locus affecting flowering with the dual function to inhibit flowering under LD conditions and promote flowering under SD conditions by regulating Ehd1 and Hd3a. Moreover, GHD8, HD1 and OsHAP5/NF-YC subunits could form a heterotrimeric complex to bind the CORE element at the promoter of $H d 3 a$ to directly regulate its expression [6]. Hd1 is a gene that was reported to genetically interact with other flowering time genes, such as Hd2/PRR37, Ghd8 (DTH8/LHD1/Hd5/ LH8), Hd6, SE5 and Ghd7 [13-23]. Some of these interactions were further validated at the molecular level, showing a complex of protein-protein interactions to regulate the expression of downstream genes. For example, HD1 and GHD7 proteins form a complex to specifically bind to a cis-regulatory region in Ehd1 and repress its expression [24, 25]. The revelation of interaction among genes at the genetic and molecular levels has considerably enhanced our understanding of the regulatory networks for flowering time.

Ghd7 and Ghd8 are two major genes identified recently with the same pleiotropic effect on the number of grains, plant height and heading date in rice. Previous results showed that a strong genetic interaction exists between $G h d 7$ and Ghd8, which seriously inhibits flowering time in the rice natural population [26]. The interaction effect could be strongly enlarged by a functional Hd1. However, it remains unknown whether and how these two genes Ghd7 and Ghd8 interact at the molecular level. To address this question, a set of near-isogenic lines (NIL) and a rice core collection panel were first used to investigate the genetic interaction effect of Ghd7 and Ghd8. Then, transcription analysis, electrophoretic mobility shift assay (EMSA) and chromatin immunoprecipitation (ChIP) assays were conducted for a likely molecular interaction. Our results revealed that Ghd8 induces the transcription of Ghd7 via GHD8-OsHAP5bHD1 complex binding to the specific CCAAT-box region in the Ghd7 promoter. Under both $\mathrm{SD}$ and $\mathrm{LD}$ conditions, Ghd8 might form a complex with OsHAP5B and HD1 activates the transcription of Ghd7 to inhibit the expression of Ehd1 and $H d 3 a$, leading to late flowering. Both functional $G h d 8$ and $G h d 7$ are pivotal for rice PS controlled by $H d 1$ and $H d 3 a$. These results regarding molecular and genetic interactions provide new insights into the gene-regulatory networks controlling flowering time and adaptation in rice.

\section{Results \\ Ghd8 acts on agronomic traits depending on functional Ghd7}

Both $G h d 8$ and Ghd7 have been identified as major effect loci, exhibiting pleiotropy in affecting heading date $(\mathrm{HD})$, plant height $(\mathrm{PH})$ and grain yield in rice $[11,27]$. The significant interaction also affected heading date [26]. To better understand how this interaction acts on different agronomic traits, we developed an $F_{2}$ population derived from a cross between near-isogenic lines NIL-Ghd8 and NIL-Ghd7 within the common Zhenshan 97B (ZS97) background. The genetic analyses showed that Ghd8 and Ghd7 interact significantly to affect HD $(p=0.005)$ and $\mathrm{PH}(p=0.026)$ in the segregating population (Fig. 1a and b). Significant difference $(p<0.001)$ in the three traits $\mathrm{HD}, \mathrm{PH}$ and spikelet number per panicle (SNP) was detected among the three genotypes at Ghd8 within the functional Ghd7 background (Fig. 1a-c). However, no significant difference was found in the assayed traits within the nonfunctional $g h d 7$ background.

The significant interaction of Ghd8 and Ghd7 was also revealed in a core collection panel consisting of 196 rice varieties. The Tukey honest test $(\alpha=0.05)$ showed that the varieties carrying Ghd8ghd7 (only Ghd8 is functional) were not significantly different in $\mathrm{HD}, \mathrm{PH}$ and SNP compared with the varieties carrying ghd8ghd7 (both alleles are loss of function) under natural LD conditions (Fig. 1d-f). However, the varieties carrying Ghd8Ghd7 showed significantly delayed HD and increased PH and SNP compared with the ghd8ghd7 and Ghd $8 g h d 7$ varieties. In particular, the varieties carrying Ghd8Ghd7 exhibited a significant delay in HD and an increase in PH compared with $g h d 8 G h d 7$. These results indicate that Ghd8 affected agronomic traits in a functional Ghd7-dependent manner. Hence, the results confirmed that Ghd8 genetically interacts with $G h d 7$ delaying flowering time and increasing plant height in both homogeneous and heterogeneous backgrounds.

\section{Interaction of Ghd8 and Ghd7 is pivotal for $\mathrm{Hd} 1$ and $\mathrm{Hd} 3 \mathrm{a}$ to control PS}

Photoperiod sensitivity is an important factor of rice cultivars to adapt to variable environments. It has been reported that $H d 1$ is a key gene that largely determines 

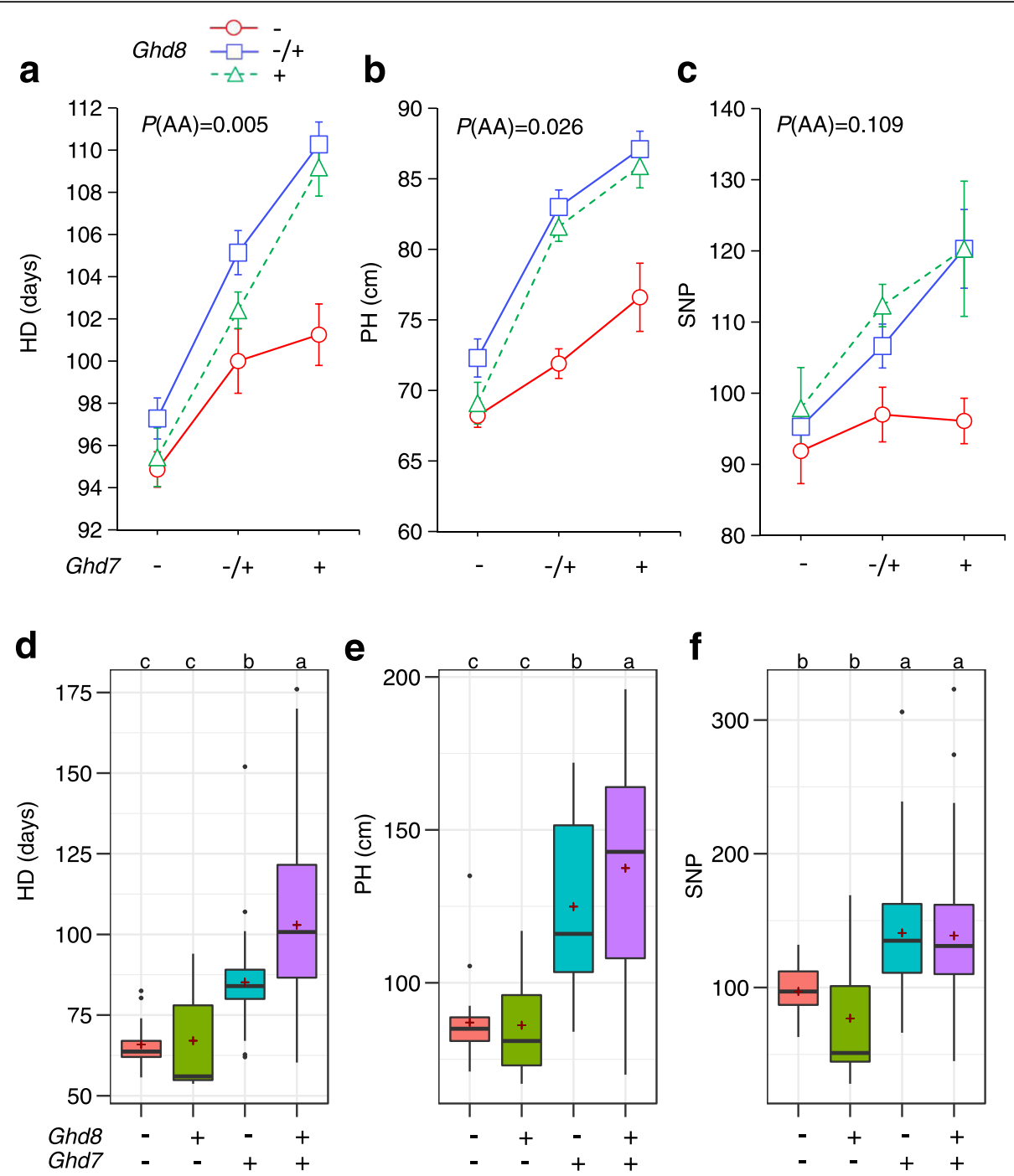

Fig. 1 Genetic interaction of Ghd7 and Ghd8 contributes to the variation of HD, PH and yield in rice. Heading date (HD, a), plant height (PH, b) and spikelet number per panicle (SNP, c) were scored in the near-isogenic line (NIL)-derived population under natural SD conditions in Hainan (N18.48, E110.02). The open red circle, blue square, and green triangle indicate nonfunctional ghd8, heterozygous, and functional Ghd8, respectively. HD(d), PH(e), and SNP (f) in four haplotypes of Ghd8 and Ghd7 in the core collection (196 accessions) grown under natural LD conditions in Wuhan (N30.52, E114.3). The different letters above the boxplots represent significance among the haplotypes at $p<0.05$ by Tukey's HSD test. $p$ values indicate the significance of the additive interaction effect between Ghd7 and Ghd8

rice PS [28]. Given that both Ghd7 and Ghd8 are involved in the Hd1-Hd3a regulatory pathway [25, 29, 30], we tested whether the interaction between Ghd7 and Ghd8 or Ghd7 and Ghd8 alone can affect the function of $H d 1$ on PS. The core collection was planted under both LD and SD conditions. The PS index of each variety was calculated by the formula $\left(\left|\mathrm{HD}^{\mathrm{LD}}-\mathrm{HD}^{\mathrm{SD}}\right|\right)$ / $\mathrm{HD}^{\mathrm{LD}}$ (Additional file 1: Table S1). A large variation in PS was observed in the rice core collection. Ghd8Ghd7Hd1 (all are functional alleles) revealed significantly higher PS on average than Ghd8Ghd7hd1 (hd1 is nonfunctional) (Fig. 2a), suggesting that $H d 1$ is the key gene determining PS variation, as reported before
[28]. Haplotype Ghd8Ghd7Hd1 also showed significantly higher PS than haplotype ghd8Ghd7Hd1, and the latter revealed a slightly higher PS that was not significant than haplotypes Ghd8ghd7Hd1 and ghd8ghd7Hd1. These results indicate that functional Ghd8 and Ghd7 and their interaction were required for the function of $H d 1$ on PS. In addition, $H d 3 a$, the other gene determining the PS of rice, also showed a similar pattern (Fig. 2b), suggesting that both functional Ghd8 and Ghd7 and their interaction played an important role in affecting the expression level of $H d 3 a$ on the control of PS. The varieties carrying functional Ghd8, Ghd7, and $H d 3 a$ promoter belonging to the high expression type [31] revealed a 

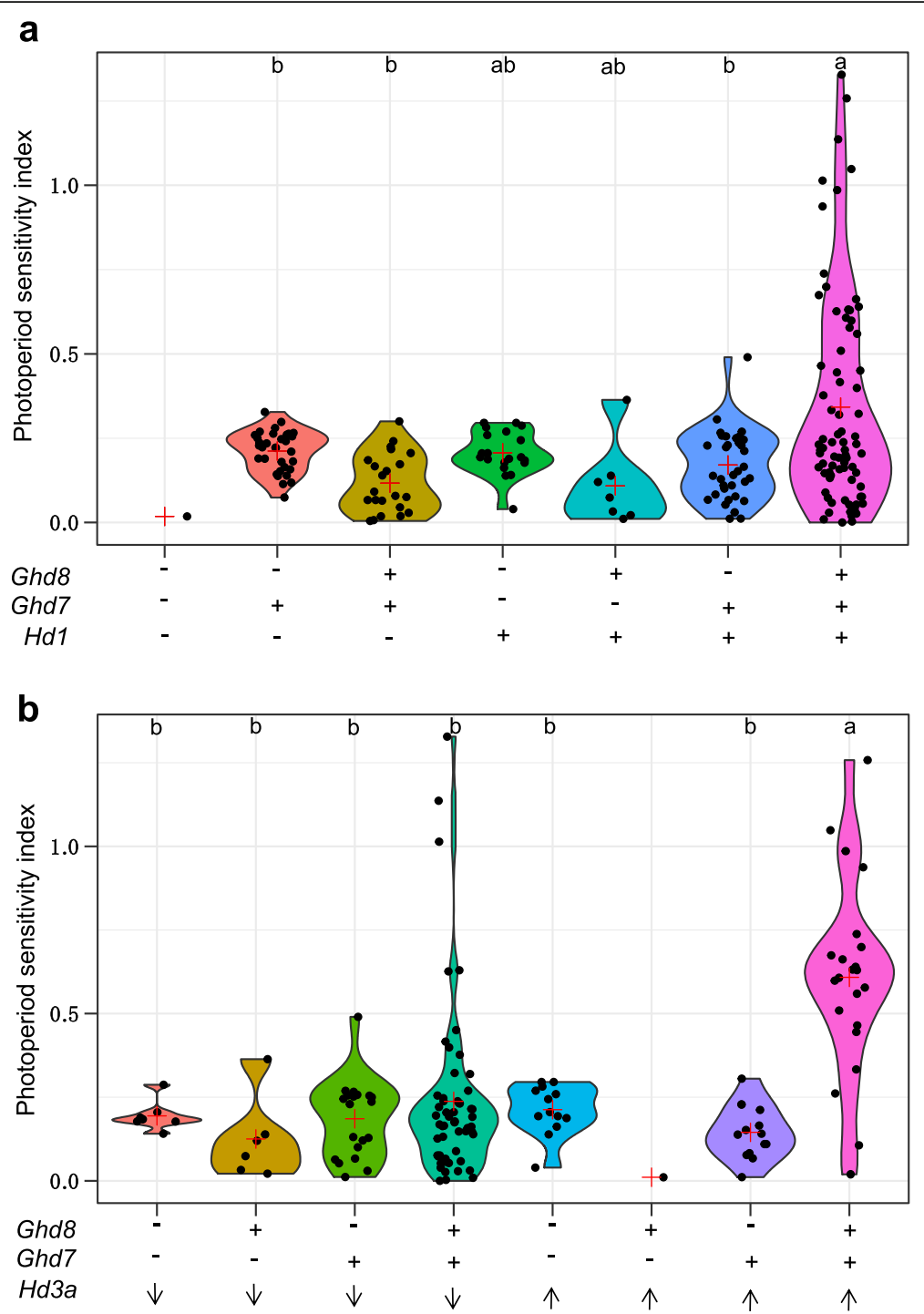

Fig. 2 Genetic interaction between Ghd7 and Ghd8 conditioned Hd1 and Hd3a affecting PS in rice. (a) Comparison of photoperiod sensitivity (PS) index among seven genotype types classed by three functional genes (Ghd7, Ghd8, Hd1) in 196 varieties as shown by the violin box.

Ghd8ghd7hd1 was not available in the core collection. (b) Comparison of PS among eight genotype types classed by two genes (Ghd7, Ghd8) and the Hd3a promoter in 139 out of the 196 varieties. The promoter types of Hd3a were selected based on the previous report [31]. The up and down arrows indicate the high and low expression levels of the Hd3a types. Fifty-seven varieties with nonfunctional hdl were not included in the (b) analysis. The red plus symbol indicates the average of PS. For Ghd8, Ghd7 and Hd1 genes, the " + " and "-" symbols at the bottom of the plots indicate the functional and nonfunctional alleles. The different letters on the top of the plots indicate the significant differences in PS among haplotypes by Tukey's HSD test $(a=0.05)$. Because there was only one variety, haplotypes ghd8ghd7hd1 and Ghd8ghd7Hd3a (high expression) were not used in the analysis

significantly higher level in the average PS than the other haplotypes. However, this significance was not observed in the varieties carrying functional Ghd8 and Ghd7 along with the promoter of $\mathrm{Hd} 3 \mathrm{a}$ belonging to the low expression type.

In addition, a segregating population segregated at three loci, Ghd7, Ghd8 and Hd1, within the homogeneous background of ZS97 was used to analyze the effects of interaction among Ghd8, Ghd7 and Hd1. The analysis of variance among genotypes showed that under natural LD conditions, the functional Hd1 (ZS97 allele) showed the strongest effect to delay HD (average $=160 \mathrm{~d}$ ) in that genotypes carrying the Ghd8Ghd7Hd1 allele combination when both Ghd7 (9311 allele) and Ghd8 (9311 allele) were functional; however, the $g h d 8 G h d 7 H d 1$ or Ghd8ghd7Hd1 genotypes carrying either single functional Ghd7 or Ghd8 exhibited earlier heading dates (Additional file 3: Figure S1). These results further indicate that the interaction between Ghd8 and Ghd7 dramatically increased the $H d 1$ effect on the control of heading date in rice. 


\section{Ghd8 upregulates Ghd7 expression to inhibit $\mathrm{Hd} 3 \mathrm{a}$}

To explore the molecular basis of the interaction, transcription levels of five flowering-related genes (Ghd8, Ghd7, $H d 1$, Ehd1 and Hd3a) were determined in 35-dayold seedling leaves of four NILs (NIL (ghd8ghd7), NIL (Ghd8ghd7), NIL (ghd8Ghd7), NIL (Ghd8Ghd7)) (Fig. 3a). NIL (ghd7ghd8) flowered at a similar time under both controlled SD (14 h dark: $10 \mathrm{~h}$ light) and LD conditions (10 h dark: $14 \mathrm{~h}$ light), showing insensitivity (PS index = 0.01 ) to the photoperiod change (Fig. 3b), although it carries the functional Hd1 alleles. NIL (Ghd8Ghd7) significantly delayed HD compared with the other three NILs (ghd8ghd7, Ghd8ghd7, and ghd8Ghd7) under both SD and LD conditions and displayed the highest PS (PS index = 0.44). In particular, NIL (Ghd8Ghd7) did not flower, even after $180 \mathrm{~d}$ under LD conditions (Fig. 3b). Compared with NIL (ghd8ghd7), NIL (ghd8Ghd7) showed no significant difference in HD under SD conditions but significantly delayed HD under LD conditions. In contrast, NIL (Ghd8ghd7) delayed HD under LD conditions while promoting HD under SD conditions, which resulted in higher PS than NIL ( $g h d 8 G h d 7)$. Relative expression analyses of the five flowering genes confirmed that the transcription level of Ghd7 was barely detected in NIL (ghd8ghd7) and NIL (Ghd8ghd7) due to the deletion of the Ghd7 gene fragment in ZS97 [11]. However, Ghd7 in NIL (Ghd8Ghd7) was expressed at a significantly higher level than that in NIL (ghd8Ghd7) under both SD and LD conditions (Fig. 3c). In addition, Ehd 1 and $H d 3 a$ expression levels in NIL (Ghd8Ghd7) showed a serious inhibition compared with that in the other NILs, which was in line with a severe delay in flowering time in NIL (Ghd8Ghd7) (Fig. 3b). The relative expression level of $H d 1$ showed no significant difference among the four NILs under both SD and LD conditions, indicating that Ghd8 and Ghd7 did not affect the transcription level of $H d 1$. These results suggest that Ghd8 might play a role in activating the transcription of the floral repressor Ghd7 to suppress the expression of the downstream genes Ehd1 and $H d 3 a$ under both LD and SD conditions, leading to late flowering.

\section{GHD8 mediated by HD1 binding to the promoter of Ghd7}

To understand how Ghd8 interacts with Ghd7 at the molecular level, we first conducted yeast two-hybrid assays for the protein interaction between GHD8 and GHD7. However, the assays did not reveal a direct interaction between them (Additional file 4: Figure S2a). It has been suggested that either Nuclear Factor A (NFYA) could interact with NF-YB/NF-YC to form the NF$\mathrm{Y}$ trimeric complex involved in transcriptional activation by binding specific cis-regulatory elements, such as the CCAAT-box or the CORE element $[6,32,33]$. To address whether Ghd8 (NF-YB) regulates the expression of Ghd7 by directly or indirectly binding the specific DNA region at the promoter of Ghd7, we investigated the CCAAT box and CORE element within the $2.5-\mathrm{kb}$ promoter region of $G h d 7$ by using the online promoter database PLACE [34]. The Ghd7 promoter region contains two CCAAT-box motifs at the proximal and distal regions: $-355 \mathrm{bp}$ and $-1603 \mathrm{bp}$ upstream of the start codon (ATG) and one CORE1-like motif CATCCACA /TGTGGATG detected at -285 bp $[35,36]$. We then developed GHD8-GFP transgenic plants and conducted ChIP assays with extracts from these transgenic lines. The overexpression GHD8-GFP transgenic line showed a significant difference in HD with an approximately $3-d$ delay compared with the wild type under natural LD conditions (Fig. 4), indicating that the GHD8-GFP protein had biological function. The precipitated products in the presence of the antibody GFP and input (no antibody) were analyzed by qPCR using a set of 9 pairwise primers (Fig. 5a, b) corresponding to different regions in the promoter region. The ChIP assays showed that the adjacent fragments $\mathrm{cp} 1$ and $\mathrm{cp} 2$ covering the CCAATbox at $-355 \mathrm{bp}$ and CORE1-like motif at $-285 \mathrm{bp}$ upstream of ATG displayed the highest enrichment in GHD8-GFP relative to that in the input control among all of the primer sets (Fig. 5b). These results indicate that the GHD8 protein might be involved in binding the CCAAT-box and/or CORE-like motif in the Ghd7 promoter to activate its transcription.

We also found that GHD8 directly interacts with OsHAP5b in both yeast two-hybrid assays and pull-down experiments (Additional file 4: Figure S2 b and c). The genetic interaction study revealed that functional Ghd8 and Ghd7 are essential for $H d 1$ controlling PS in rice (Fig. 2, Additional file 3: Figure S1), implying these three genes may work together to regulate flowering time. To test if the complex formed by GHD8 and OsHAP5b recognizes the CCAAT-box at the promoter of Ghd7 by interacting with HD1, EMSA was performed by using the F1 probe that overlaps with the cp1 and cp2 fragments (Fig. 5a, c). The EMSA results revealed that the recombinant HD1 (MBPHD1) alone could directly bind to the F1 fragment in vitro with a weak binding ability, and the excess unlabeled F1 fragment (competitor) inhibited the binding (Fig. 5c, d). We found that MBP-HD1 incubated either with His-GHD8 or with His-GHD8 and His-HAP5b could bind to the F1 fragment with different affinities in the EMSA assay (Fig. 5d). Moreover, a considerably stronger binding signal was observed when GHD8, HD1 and OsHAP5b proteins were incubated together with probe F1 (Fig. 5d), which is consistent with a previous study demonstrating that HD1 interacts with GHD8 and OsHAP5b to form a trimeric complex to bind the CORE motif in rice [30]. This result was also confirmed by using the competition assays with the unlabeled F1 fragment and the probe MF1 (a mutated F1 probe lacking the CCAAT element) (Fig. 5e). 
a
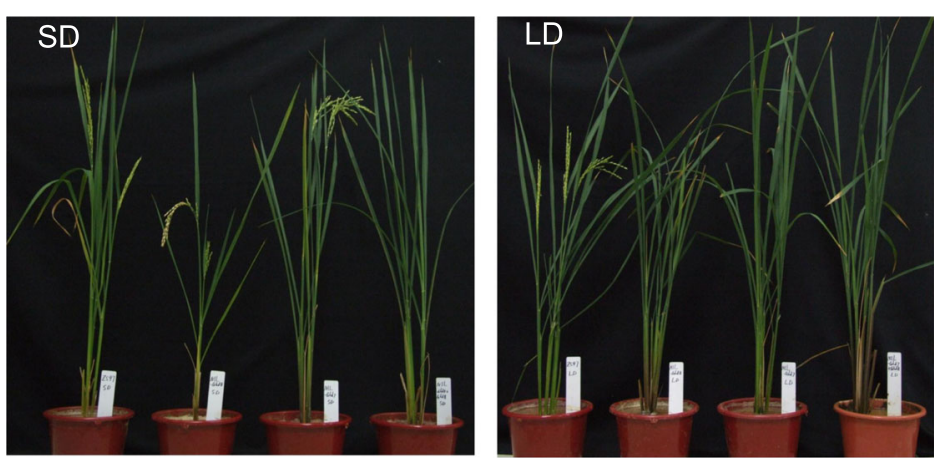

Ghd8

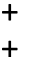

$\begin{array}{lllll}- & + & - & + \\ - & - & - & + & \end{array}$

b

$\square$ SD $\square$ LD $\square$ PS

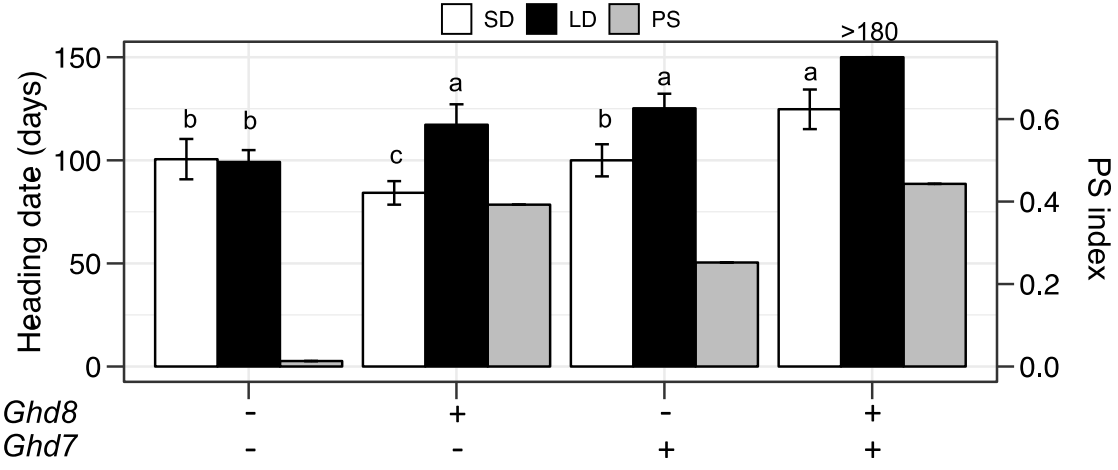

C
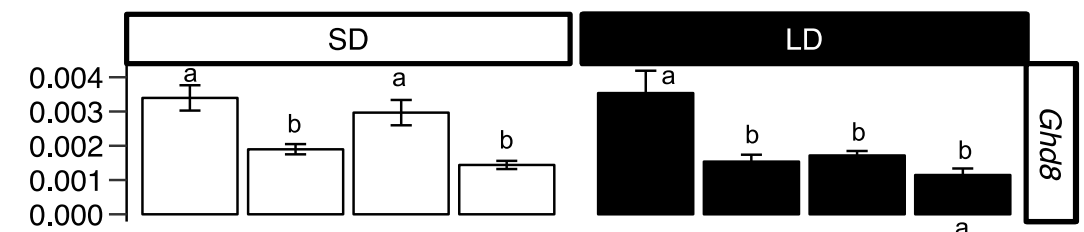

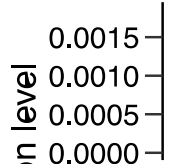

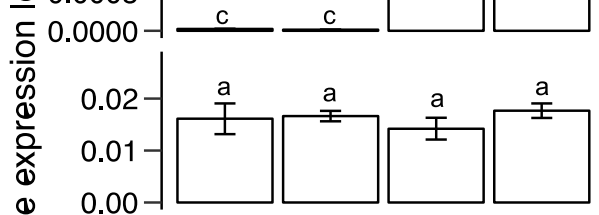

$\frac{1}{\frac{\pi}{10}}$
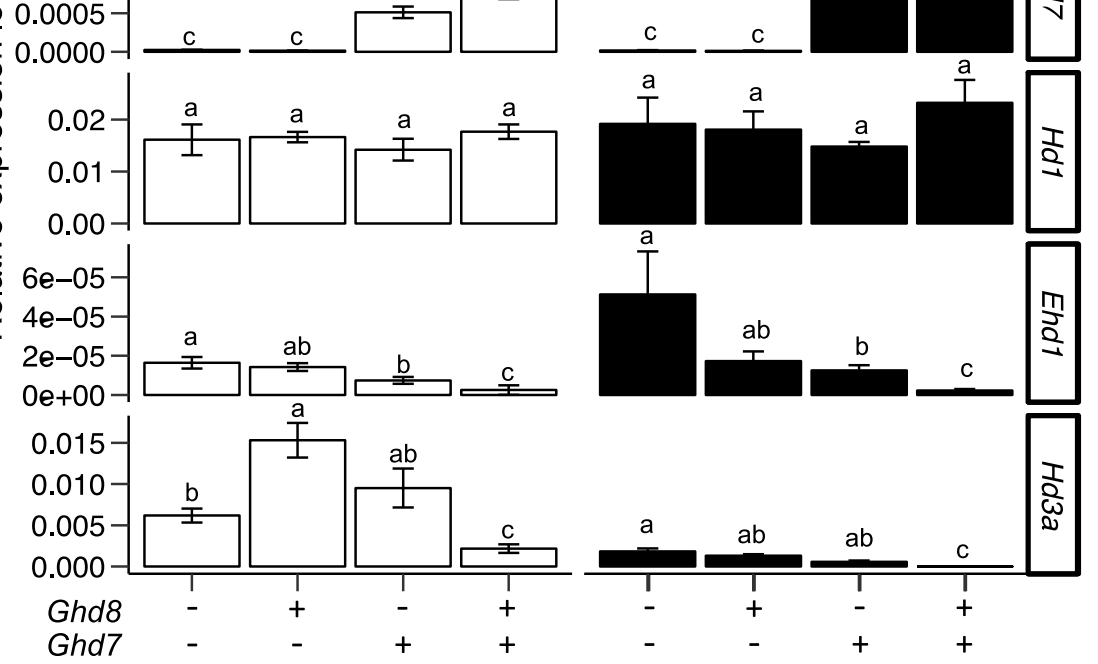

Fig. 3 (See legend on next page.) 
(See figure on previous page.)

Fig. 3 Expression levels of key flowering genes in the NILs with different combinations of Ghd7 and Ghd8 alleles. (a) Performance on the heading date of four NILs in the growth chamber under SD (10 h L: $14 \mathrm{~h} \mathrm{D}$ ) and LD conditions (14 h L: $10 \mathrm{~h} \mathrm{D})$. (b) Heading date of NIL (ghd8ghd7), NIL (Ghd8ghd7), NIL (Ghd7ghd8) and NIL (Ghd8Ghd7) under SD (white bars) and LD conditions (black bars), and photoperiod sensitivity (PS) index (gray bars). (c) Expression levels of Ghd8, Ghd7, Ehd1, Hd3a and Hd1 under SD and LD conditions. The y-axis indicates the relative expression level of the gene normalized by Ubiquitin. Tukey's HSD test was used to test the significance in heading date or expression among the four haplotypes under SD or LD conditions. The different letters above the bars denote the significance at $p<0.05$. Mean \pm SE $(n=4-6)$

\section{Discussion}

\section{Molecular interaction of Ghd8 and Ghd7}

Ghd7 and Ghd8 have been reported as the major genes determining flowering time and plant growth [11]. In the present study, we demonstrate that Ghd8 genetically interacts with Ghd7, delaying heading date in near-isogenic lines and in the rice core collection. Importantly, we found that the transcript level of Ghd7 was activated by Ghd8 through the GHD8HAP5b-HD1 complex.

Under LD conditions, Ghd7 strongly represses the expression of Ehd1, leading to decreased expression of florigen $H d 3 a$ to inhibit flowering in rice [11]. Recent studies have revealed that Ghd7 is controlled at the transcriptional and posttranslational levels by other flowering time genes, such as OsELF3 and Hd16 [29, 37], which suggest that modification of Ghd7 is a critical role in the decision of flowering time in the long-day repression pathway. A regulatory pathway in a previous report showed that $H d 1$ upregulated the expression level of Ghd7, leading to downregulation of Ehd1 and $H d 3 a$ under LD conditions [24]. Additionally, Nemoto et al. reported that the interaction between HD1 and GHD7 proteins resulted in a synergistic repression of Ehd1 to cause a serious delay of flowering time [25]. The GHD7-HD1 complex represses Ehd1 by binding to a cis-regulatory region of Ehd1 [6], which suggested that $H d 1$ was integrated into the LD repression pathway. Due to the high expression of Ghd7, Ehd 1 and $H d 3 a$ were repressed to delay flowering [27, 30].
Hence, Ghd7 not only works as a signal integrator to receive the output from circadian clock [37], but also plays a role in transmitting regulation signals of other floweringrelated genes, such as $H d 1$ to Ehd1, to affect flowering time. It is notable that no difference in the $H d 3 a$ expression level was revealed in the NILs ghd8Ghd7 and ghd8ghd7; intriguingly, $\mathrm{Hd} 3 \mathrm{a}$ was repressed in NIL (Ghd8Ghd7) by approximately eight-fold compared with that in NIL (Ghd8ghd7) under SD conditions (Fig. 3c). These results suggest that $G h d 7$ did play a role as a repressor of the expression of $H d 3 a$ in a dependent manner of functional Ghd8 (Fig. 6). Furthermore, our data revealed the enhanced effect of Ghd8 and Ghd7 interaction on flowering time in the genetic background of ZS97 with a functional $H d 1$; however, the transcription level of $H d 1$ remains unchanged in the NILs with the ZS97 background. This finding suggests that Ghd8 might integrate the Hd1-Ghd7 pathway in the regulation of rice flowering through a posttranscriptional mechanism that the protein GHD8 or GHD7 might interact with HD1.

Previously, Ghd8 was shown to play a similar role as Hd1 with repression or activation in flowering time under LD or SD conditions [27, 30]. Hd1 repressed $H d 3 a$ under LD conditions or activated gene expression under SD conditions in the presence of the nonfunctional $g h d 7$ [25]. In the current study, the dual function for Ghd8 on flowering was also found to be dependent on the presence of the nonfunctional ghd7 (Fig. 3b, c). In the comparison between NIL (ghd8ghd7) and NIL
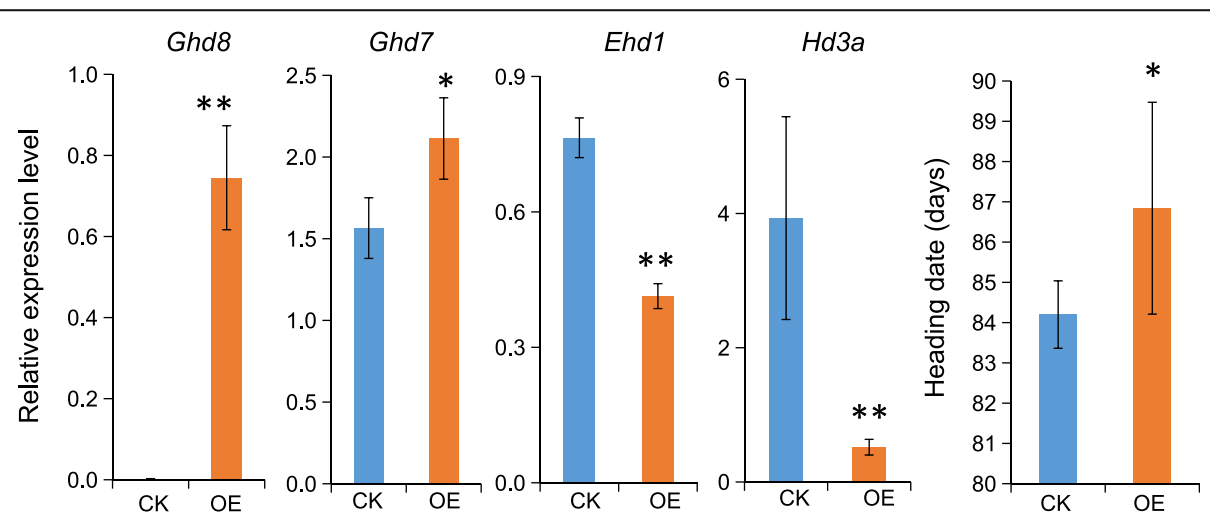

Fig. 4 qRT-PCR analysis of several flowering-related genes in the Ghd8 overexpression line. Leaves were harvested from 35-day-old seedlings of the Ubi::GHD8:GFP overexpression line (OE) and its corresponding negative control (CK) grown under natural LD conditions. Single or double asterisks denote significant differences at $P<0.05$ or $P<0.01$ by $t$-test 


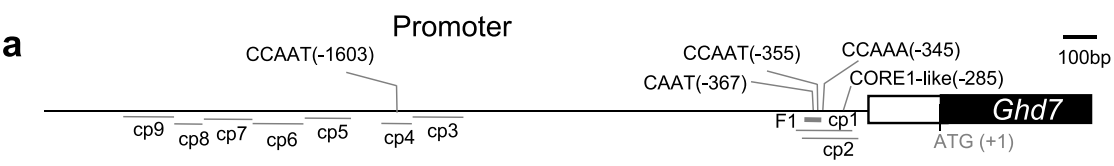

\section{b}
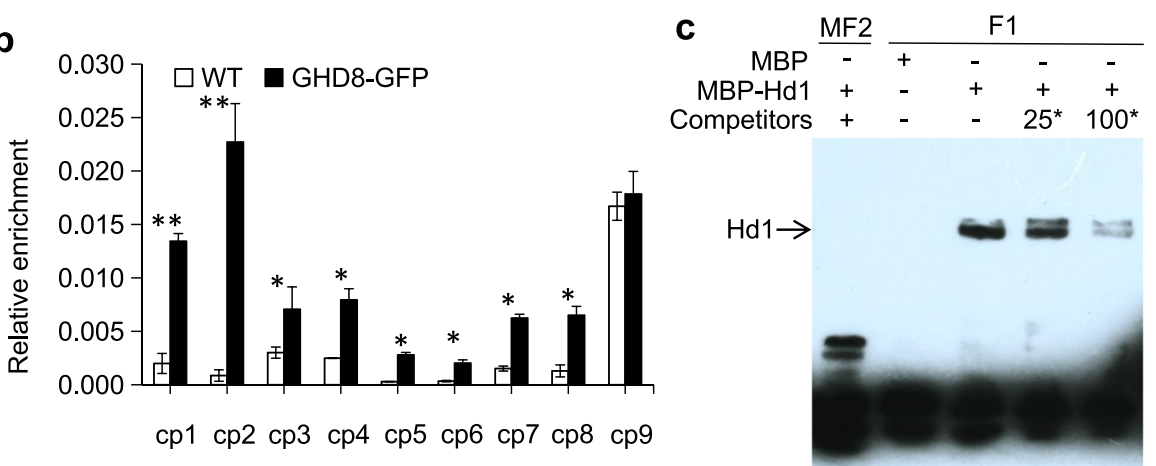

d

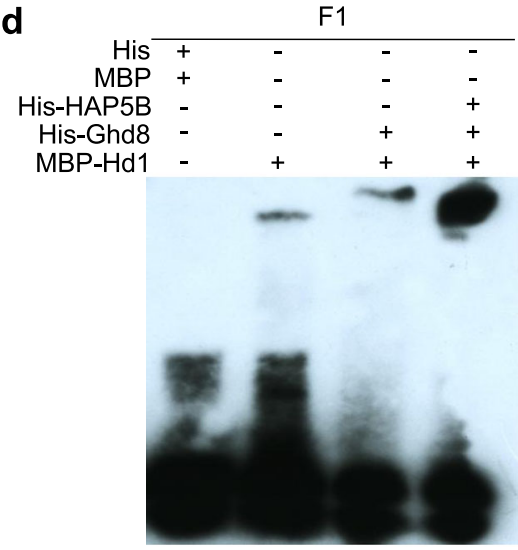

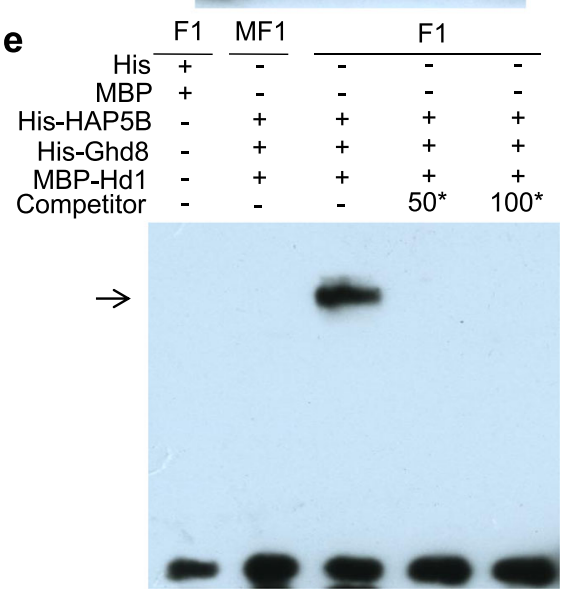

e

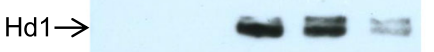

$\operatorname{Hd} 1 \rightarrow$
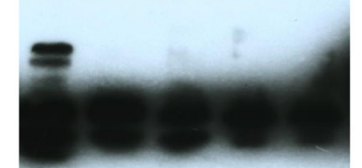

MF1

$\begin{array}{ccc}- & - & - \\ + & + & + \\ + & + & + \\ + & + & + \\ - & 50^{*} & 100^{*}\end{array}$

\section{-}

Fig. 5 GHD8 binds to the promoter of Ghd7. a A schematic diagram of the Ghd7 gene model showing the locations of two CCAAT-box motifs and one CORE1-like motif from the start codon ATG. The relative positions of the fragments from cp1 to cp9 used for the ChIP assay are given below the gene model; F1 indicates the fragment covering the CCAAT-box motif for the gel shift assays. $\mathbf{b}$ ChIP analysis of GHD8-GFP fusion protein using an anti-GFP antibody. The precipitated chromatin fragments were analyzed using the 9 primer sets for the target regions (cp1 to cp9) shown in (a). c Gel shift assays. Recombinant HD1 protein was incubated with biotin-F1 in the absence or presence of 25- or 100-fold molar excess of the unlabeled probe as the competitor. The arrow indicates the shifted band of HD1 protein. Maltose-binding protein (MBP) and His (polyhistidine)-tag protein were used as a negative control. d GHD8 interacts HD1 to binding F1 probe. e Interaction of the complex GHD8OsHAP5b-HD1 (as shown by arrow) and F1 probe in the absence or presence of 50- or 100-fold molar excess of the unlabeled probe. The mutant F1 (MF1 or MF2) probe lacking the CCAAT box as a negative control

(Ghd8ghd7) with the functional $H d 1$ background, Ghd8 caused early flowering under SD conditions. Consistently, the $H d 3 a$ expression was much more activated in NIL (Ghd8ghd7) than that in NIL (ghd8ghd7). Previous study also revealed that under SD conditions, $H d 1$ could activate the expression of Ehd1 and $H d 3 a$ regardless of Ghd7 functions [11]. In addition, it was reported that HD1-containing NF-Y complex has the capacity to bind a conserved response element in the $H d 3 a$ promoter [6]. These results suggest that $G h d 8$ acts as an activator and might work together with $H d 1$ to regulate $H d 3 a$ under SD conditions (Fig. 3). As mentioned above, the GHD7HD1 complex could bind the Ehd1 promoter under LD conditions [25]. The GHD8-HD1 complex promoted the flowering time through regulating $H d 3 a$ expression [30]. And GHD8-HAP5b-HD1 complex may activate Ghd7 expression to enhance its repression effect on flowering time. These data point to the flowering regulatory pathway linking Ghd8 to $\mathrm{Hd} 1$ and Ghd7. Taken together, these results indicate that $H d 1$ not only plays an important role in activating transcription under SD conditions but also incorporates GHD7 protein, inhibiting the expression of Ehd1 in the LD repression pathway. Moreover, Ghd8 could enhance the inhibitory role of Ghd7 in flowering time under both SD and LD conditions (Fig. 3c). These findings suggest that the $H d 1$ 

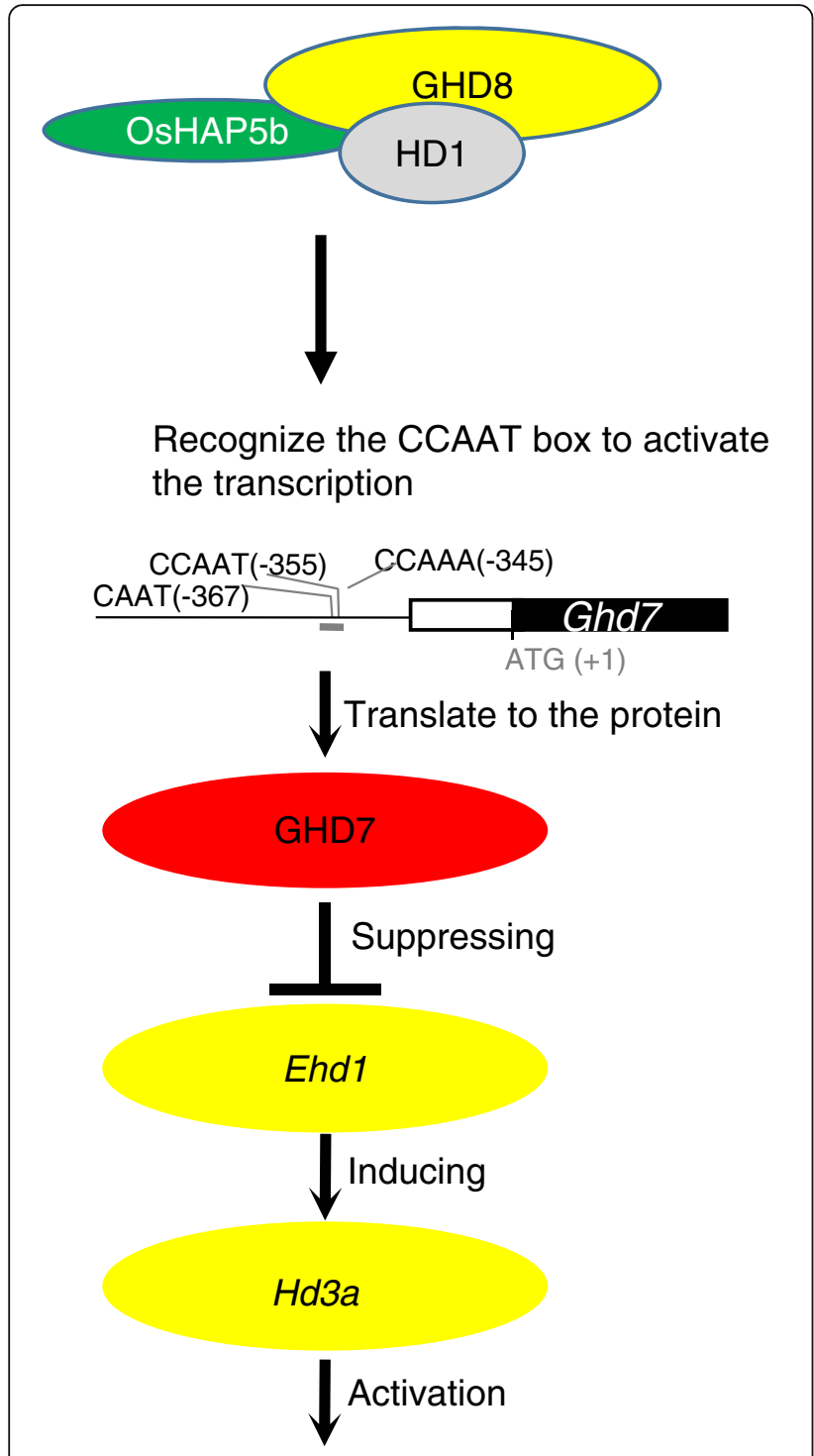

Flowering

Fig. 6 Model of GHD8 complex regulating flowering time. The heterotrimer GHD8-OsHAP5b-HD1 targets the CCAAT-box element and/or CORE motif of the Ghd7 promoter to activate its expression, leading to suppression of Ehd1 and downregulation of Hd3a expression

function become flexible to affect flowering time in terms of the photoperiod change through interacting with other transcription factors like Ghd8 and Ghd7.

\section{GHD8-OsHAP5b-HD1 binds the CCAAT-box}

Ghd8 encodes a homolog of HAP3 or NF-YB subunit, together with HAP2 and HAP5 or NF-YA and NF-YC, constitutes the trimeric complex to bind the CCAATbox at the promoter to activate the gene transcription
[33]. However, in the absence of NF-YA, other transcription factors, such as CO or bZIP, also interact with NF$\mathrm{YB}$ and NF-YC to form new complexes, leading to different binding sites that do not strictly bind to the CCAAT-box [30, 38-41]. For example, the CCT domain of $\mathrm{CO}$ and $\mathrm{CO}$-like protein could interact with the complex of NF-YB and NF-YC to bind the CORE-element in Arabidopsis $[35,42]$. In rice, the GHD8-OsHAP5b-HD1 complex has been reported to directly bind the CORE1 element on the promoter of $\mathrm{Hd} 3 \mathrm{a}$ to regulate flowering [30]. In the current study, GHD8-OsHAP5b was found to interact with HD1 to form a trimeric complex to bind the CCAAT-box at the $-355 \mathrm{bp}$ site of the Ghd7 promoter. HD1 alone or the HD1-Ghd8 dimer were able to directly bind to the DNA fragment F1 that harbors the CCAAT-box, but this fragment did not cover the CORE1-like motif sequences (Fig. 5). Therefore, we propose that GHD8-HD1 or HD1 protein can recognize the CCAAT motif at the -355 bp site.

In addition, the trimeric complex of NF-YB-NF-YC-CO appears more important to the binding of the CORE motif in Arabidopsis [35], and even mutation of any one of them can completely eliminate the binding ability of the complex. In contrast, the NF-YB1 subunit alone was reported to directly bind downstream genes without the assistance of other NF-Ys in the endosperm in rice [40,43]. Our study revealed the low binding affinity of HD1 and HD1GHD8 dimer on the CCAAT-box motif, suggesting that HD1 protein might be able to bind both the CCAAT-box motif and the CORE1 motif and have an overlapping function with OsHAP2/NF-YA and compete with these subunits to recognize the CCAAT-box motif [44]. Although the role of the CORE1-like motif in the binding stability of the GHD8-OsHAP5b-HD1 complex needs to be further investigated, the molecular interaction between Ghd8 and Ghd7 to control of $H d 3 a$ expression supports that the GHD8-OsHAP5b-HD1 regulatory complex is involved in the Ghd7 repression pathway. These results suggest the existence of multiple targets for the GHD8OsHAP5b-HD1 complex that fine tune the spatiotemporal regulation of gene expression in the flowering network, which might have a greatly contributed to the adaption of rice to various cultivation areas.

\section{Implications of Ghd7 and Ghd8 interaction}

Asian cultivated rice has two subspecies: indica and japonica, both of which are distributed in different ecogeographic environments [31]. In the present study, Ghd8 and Ghd7, as well as their interaction effect, were detected in response to the specific photoperiod conditions. The varieties with both weak functional $G h d 7$ and Ghd8 alleles dominate in the northern region between $35^{\circ} \mathrm{N}$ and $45^{\circ} \mathrm{N}$, where farmers usually practice single 
planting in the summer cultivation season of long daylength (Additional file 5: Figure S3). The majority of varieties planted there are japonica subspecies [11, 27] and show a relatively low PS (average PS index $=0.2$ ) (Additional file 1: Table S1). In parallel, the varieties with strong functional Ghd7 and Ghd8 with a relatively high PS are mainly found in subtropical and tropical regions between $25^{\circ} \mathrm{N}$ and $15^{\circ} \mathrm{N}$. Additionally, a portion of varieties that contain both nonfunctional alleles $g h d 7$ and $g h d 8$ occur predominantly along $25^{\circ} \mathrm{N}$ latitudes (Additional file 5: Figure S3). This finding suggests that nonfunctional $g h d 7$ and $g h d 8$ are beneficial for the rapid growth of varieties required in this zone, where multiple plantings occur.

Notably, almost all of the northern japonica varieties carrying both functional alleles of Ghd7 and Ghd8 have functional $\mathrm{Hd} 1$ (Additional file 5: Figure S3). However, these varieties flowered early $(68-85 \mathrm{~d}$ ) in natural LD conditions. This unexpected observation is different from the late heading in the NILs with the indica background, although they carry all functional alleles of Ghd7, Ghd8 and Hd1. One explanation is that the japonica varieties might have divergent Ghd7, Ghd8 or $H d 1$ alleles with a reduced/weaker function that probably facilitates japonica rice adaptation to the northern/high latitudes [11, 27, 31, 45, 46]. Our study indicates that a high disequilibrium linkage of Ghd7 and Ghd8 exists in japonica rice $(p<0.01)$, and Ghd8 or Ghd7 also occurs in linkage disequilibrium with $H d 1$ and/or $H d 3 a$ in either indica or japonica. This high linkage relationship among these genes suggests that some preferable genes in the regulation network might be selected together for suitable flowering (Additional file 6: Figure S4). The other possible explanation is that some flowering gene interaction unidentified in rice helps to counteract severely delayed flowering that arose by functional Ghd7, Ghd8 and $H d 1$ [23, 29].

There is a significant association between flowering time and grain yield in rice. A longer flowering time is usually associated with higher grain yield. However, there is a trade-off between increased yield potential and delays in HD. Therefore, in terms of the action of Ghd7 and Ghd8, which play a crucial role in the regulation of flowering, these genes should be considered together in high-yield breeding programs, especially for those varieties used in the low latitude regions, where multiple plantings are commonly applied for obtaining high yield per area. As rice is the main source of daily calories, deciphering the combined effects of Ghd8, Ghd7, and $H d 1$ in flowering regulatory pathways will help elucidate the adaptation mechanism to various photoperiod conditions and will be beneficial in molecular design for the development of rice varieties with high yield potential.

\section{Conclusions}

This study investigates the genetic interaction effect between two major genes, Ghd8 and Ghd7, by using the segregating population generated from the cross between NIL lines and a natural germplasm panel in rice. The molecular basis of their interaction was further identified by using a series of experiments, including EMSA and ChIP. We found that Ghd8 regulates transcript of Ghd7 through the GHD8-OsHAP5b-HD1 regulatory complex. Thus Ghd8 and Ghd7 together with $H d 1$ largely explain seriously delayed flowering time, increased plant height, and improved yield potential in rice varieties. We also found that both functional Ghd8 and Ghd7 are pivotal for rice PS controlled by $H d 1$ and $H d 3 a$, which is highly important for rice varieties to adapt to different environments. These findings provide insights into the molecular and genetic bases of the gene interaction on crop productivity and adaptation and reveal that the selectivity and combination of different functional alleles could be highly useful for improving yield potential in rice breeding programs.

\section{Methods}

\section{Plant material and growth conditions}

The panel of 196 rice accessions was used for nucleotide sequencing analysis $[47,48]$. These accessions were obtained from China National Rice Research Institute and the International Rice Research Institutes. The varieties were grown in Wuhan (N30.52, E114.3) under natural LD conditions and in Hainan (N18.48, E110.02) under natural SD conditions for the measurements.

Two advanced backcross lines harboring the genomic regions with either functional Ghd7, Ghd8 or $H d 1$ were used to generate $F_{1}$ hybrids [20]. The $F_{1}$ plants were then backcrossed with ZS97 twice to create the $\mathrm{BC}_{2} \mathrm{~F}_{1}$ generation. The segregating population of $\mathrm{BC}_{2} \mathrm{~F}_{2}$ containing approximately 200 individuals was grown in Hainan. The SSR markers RM5436, PID2, and RM121 were used to select the heterozygous segments at Ghd7, Ghd8 and $H d 1$ during the crossing scheme. The four homozygous genotypes of Ghd8Ghd7, Ghd8ghd7, ghd8Ghd7 and ghd8ghd7 with the common background of ZS97 were selected as the near-isogenic lines (NILs) in which $H d 1$ is functional.

To compare the HD and PH of the NILs under different light/dark conditions, 10 plants for each NIL were grown in LD (14 h light: $10 \mathrm{~h}$ dark) and SD (10 h light: $14 \mathrm{~h}$ dark) conditions in growth chambers. Flowering time was scored from 8 individuals per line. HD was defined as the time when the first panicle appeared from the flag leaf.

\section{DNA sequencing}

The rice core collection was genotyped by sequencing at the coding region of Ghd7, $H d 1$, Ehd1, $H d 3 a$ and Ghd8 
covering the functional site described in recent papers $[11,12,27]$. The PCR amplified fragments were sequenced directly using BigDye Terminator Cycle Sequencing v3.1 (Applied Biosystems, Foster, USA) after digestion and purification according to the manufacturer's specifications. The primers used are listed in Additional file 2: Table S2.

\section{Gene expression analyses}

Leaves from the main culm of NILs of 35-day-old plants were harvested to analyze the transcription levels of Ghd8, Ghd7, Hd3a, Ehd1 and Hd1. The NILs were grown in the growth chambers respectively under SD and LD conditions at $30{ }^{\circ} \mathrm{C}$ in the light and $26^{\circ} \mathrm{C}$ in the dark. Samples were collected at Zeitgeber time (ZT) 4 under SD conditions and at ZT 8 under LD for gene expression analysis. ZT 0 indicates dawn. The samples were collected for RNA extraction using an RNA extraction kit (TRIzol Reagent, Invitrogen). The time points for sampling corresponded to the expression peak for each gene according to the reports [11,27]. Approximately $2 \mu \mathrm{l}$ of RNA was used to synthesize the cDNA in a $20 \mu \mathrm{l}$ reaction volume by using SuperScriptII reverse transcriptase (Invitrogen) after treatment with DNase I to remove the contaminating genomic DNA. Real-time PCR (RT-PCR) was performed in a total volume of $25 \mu \mathrm{l}$ on an Applied Biosystems 7500 RealTime PCR System, which contained $2 \mu \mathrm{l} \mathrm{cDNA}, 2 \mu \mathrm{l}$ primers $(0.4 \mu \mathrm{M}), 8.5 \mu \mathrm{l}$ distilled water and $12.5 \mu \mathrm{l}$ of SYBR $^{\circ}$ Green PCR Master Mix (Applied Biosystems) for each reaction. The expression data were normalized by using the ubiquitin gene with the relative quantification method [49]. All experiments were conducted in at least three biological and three technical replicates. The primers used for the transcription analyses are listed in Additional file 2: Table S2.

\section{Yeast two-hybrid assays}

The protein-coding regions of Ghd7, Ghd8 and OsHAP5b were amplified using gene-specific primers with added restriction enzyme sites, respectively (Additional file 2: Table S2). Then, the Ghd7 or OsHAP5b product amplified by PCR was fused into the activation domain (AD) vector pGADT7 as a prey system with EcoRI and XhoI sites, and the Ghd8 product with $E c o R I$ and $B a m H I$ sites was fused to the DNA-binding domain (BD) vector pGBKT7 as bait system. All constructs were verified by sequencing. The cotransformation of two plasmids carrying the Ghd8, OsHAP5b and Ghd7 genes into the AH109 yeast strain and cell culture were performed according to the manufacturer's protocols (Matchmaker Gold Yeast Two-Hybrid System, Clontech). Constructs of pGBKT7-53 (pBD-53) and pGADT7-T (pAD-T) served as positive controls, and constructs of pGBKT7-Lam and pGADT7-T (pAD-T) served as negative controls.

\section{In vitro pull-down assay}

The coding region of Ghd8 was cloned into the pET-32a vector (Novagen) and pGEX-6P-1 vector (GE Healthcare) with EcoRI and XhoI sites, respectively. To obtain the OsHAP5b protein, we used the same method for GHD8. The recombinant expression vector was expressed in Escherichia coli Transetta (DE3) cells (Transgen). The pull-down experiment was performed as described previously [50]. In brief, supernatants with equal amounts of Glutathione S-transferase (GST) or OsHAP5b-GST with GHD8-His recombinant proteins were incubated for $6 \mathrm{~h}$ at $4{ }^{\circ} \mathrm{C}$ in a total volume of $2 \mathrm{ml}$ of pull-down buffer $(20$ mmol Tris- $\mathrm{HCl}, \mathrm{pH} 8.0,200 \mathrm{mmol} \mathrm{NaCl}, 1 \mathrm{mmol}$ EDTA, $0.5 \%$ Lgepal CA-630 and protease inhibitor), after which $200 \mu \mathrm{l}$ of GST resin was added (GE Healthcare; 17-513201 ) and the mixture incubated for $2 \mathrm{~h}$ at $4{ }^{\circ} \mathrm{C}$. The binding reaction was then washed 5 times $(10 \mathrm{~min}$ each time at $4{ }^{\circ} \mathrm{C}$ ) using the pull-down buffer. After extensive washing, the pulled-down proteins were eluted by boiling at $95^{\circ} \mathrm{C}$ for $10 \mathrm{~min}$, separated on 12\% SDS-PAGE and detected by immunoblots using an anti-GST antibody (Abcam; ab19256) and anti-His antibody (Abcam; ab9108), respectively.

\section{ChIP (chromatin immunoprecipitation)}

For ChIP assays, wild-type and GHD8-GFP transgenic lines were used for chromatin extraction and immunoprecipitation following the method described [51]. Briefly, young leaves from approximately 35-day-old seedlings were collected under LD conditions ( $15 \mathrm{~h}$ light: $9 \mathrm{~h}$ dark). After treated with formaldehyde to cross-link the proteins to the DNA, the cross-linked lysate was sonicated to shear DNA to the smaller fragment size. The soluble chromatin fragments were isolated and purified for the following steps. Immunoprecipitation with anti-GFP (Abcam; ab290) was performed for wild-type and GHD8-GFP transgenic lines with 3 repeats. The precipitated DNA was analyzed by quantitative RT-PCR using specific primer sets listed in Additional file 2: Table S2, designed to cover the CCAAT-box element within a 2-kb primer region upstream of ATG of Ghd7.

To construct the GHD8-GFP fusion, maize (Zea mays) ubiquitin promoter-GFP cassette from pU1301 was used to substitute the GUS fragment of pCAMBIA1391Xb to create a modified pCAMBIA1391Xb [51]. The conservative domain within the Ghd8 coding region involving in the binding and interaction function was amplified and inserted into the KpnI and BamHI sites of the modified pCAMBIA1391Xb to obtain the construct Ubi::GHD8GFP for rice transformation. The Agrobacterium mediated genetic transformation was used to generate the 
transgenic plants with Ubi::GHD8-GFP construct in the Nipponbare background.

\section{Electrophoretic mobility shift assay (EMSA)}

The pET32-based expression vectors for GHD8 and OsHAP5b were used to express recombinant proteins fused to a thioredoxin and a His (polyhistidine)-tag in tandem at their N-terminal ends. An E. coli BL21 strain (Transgen) was transformed with vectors and grown at $37^{\circ} \mathrm{C}$. pMAL vector (New England Biolabs, E8000S) was used to obtain the recombinant HD1 proteins with the maltose-binding protein (MBP) at the down-stream. The recombinant proteins pET32-GHD8, pET32-OsHAP5b and pMAL-c2X-HD1 were purified with Ni-NTA agarose (QIAGEN; No. 30210) and amylose resin beads (New England Biolabs) respectively. The Ghd7 promoter fragment F1 (including the putative binding site CCAAT-box) were produced by annealing of 3'-biotinlabeled oligonucleotides Ghd7F/R (Sangon Biotech), respectively. DNA binding reactions were performed in the presence or absence of unlabeled F1 and labeled mutated F1 (MF1 and MF2) fragments at room temperature for $20 \mathrm{~min}$ in $5 \mathrm{mM}$ Tris, pH 7.5, $25 \mathrm{mM} \mathrm{KCl}, 0.5 \mathrm{mM}$ DTT, $5 \mathrm{mM} \mathrm{MgCl} 2,2.5 \%$ glycerol, $0.05 \%$ NP-40 and 50 $\mathrm{ng} / \mathrm{uL}$ poly $(\mathrm{dI}-\mathrm{dC})$. We followed the protocol from the LightShift Chemiluminescent EMSA Kit (Thermo; No.20148), and the samples were run on 5\% polyacrylamide gels. The primers used for EMSA are listed in Additional file 2: Table S2.

\section{Linkage disequilibrium analysis}

Linkage disequilibrium analysis of Ghd7, Ghd8, OsHAP5b, $H d 1$ and $H d 3 a$ was performed using the software TASSEL 3.0.147 (http://www.maizegenetics.net/). The sequences including single nucleotide polymorphisms in the promoter region ( $1.5 \mathrm{~kb}$ upstream of ATG), coding region and 3'UTR ( $1 \mathrm{~kb}$ downstream of the stop codon) of these genes in 532 varieties were obtained from the website (http://ricevarmap.ncpgr.cn/django/snp_id/). All SNPs in each gene from the varieties were input into TASSEL to conduct linkage disequilibrium analysis. Out of 532 varieties, 2 groups, each including 295 indica and 156 japonica were also separated for linkage disequilibrium analysis, and a threshold of $1 \%$ was set to filter the SNPs with low frequencies.

\section{Statistical analysis}

Analysis of variance (ANOVA) of gene interaction on the assayed traits in the $\mathrm{F}_{2}$ segregating population derived from the cross of NIL $(G h d 7) \times$ NIL $(G h d 8)$ was conducted using Statistica software [52]. Tukey's HSD (honest significant difference) test in JMP software was employed to determine which groups were significantly different [53].

\section{Supplementary information}

Supplementary information accompanies this paper at https://doi.org/10. 1186/s12870-019-2053-y.

Additional file 1. Table S1. Functional alleles of Ghd8, Ghd7 and Hd1 and promoter type of $\mathrm{Hd} 3 \mathrm{a}$ in the panel of 196 rice varieties.

Additional file 2. Table S2. PCR primers and EMSA probes used in this study.

Additional file 3. Figure S1. Heading date (HD) in the genetic population segregated at Ghd8, Hd1 and Ghd7. Ghd8, Hd1 and Ghd7 indicate the functional alleles, and lowercase ghd8, hdl and ghd7 indicate the nonfunctional alleles. Ghd8/ghd8, Ghd7/ghd7 and Hd1/hd1 indicate heterozygote.

Additional file 4. Figure S2. Detection of protein interaction. (a) Yeast two-hybrid assays for GHD8 and GHD7 interaction. (b) Yeast two-hybrid assays for GHD8 and OsHAP5b. (c) Pull-down assay for GHD8 and OsHAP5b in vitro.

Additional file 5. Figure S3. Geographic distribution of 196 rice varieties. All 196 rice varieties were highlighted by four combinations of Ghd8 and Ghd7 and subdivided into indica or japonica subspecies. The stacked bar graph indicates the distribution and frequency of four combinations (haplotypes) of Ghd7 and Ghd8 alleles. The map image was taken from Wikimedia Commons: https://commons.m.wikimedia.org/wiki/File.

Additional file 6. Figure S4. Linkage disequilibrium patterns among Ghd8 and Ghd7 and the other related flowering genes $\mathrm{Hd}$, $\mathrm{Hd} 3 \mathrm{a}$ and OsHAP5b in 532 rice varieties. Total population, indica and japonica subgroups individually were used for the analysis.

\section{Abbreviations}

ChIP: Chromatin immunoprecipitation; EMSA: Electrophoretic mobility shift assay; HAP: Heme activator protein; HD: Heading date; LD: Long-day conditions; NF-Y: Nuclear transcription factor Y; NIL: Near isogenic lines; PS: Photoperiod sensitivity; QTL: Quantitative trait locus; SD: Short-day conditions

\section{Acknowledgements}

We thank Dr. Daniel Schachtman for critical reading of the manuscript. We thank Dr. Chongrong Wang, Dr. Fenyi Hu and Ms. Chengju Tan for heading data collection of rice germplasms. We are grateful to Dr. Yueming Guo for assistance in data analysis. We thank Jian Yu for assistance on field management.

\section{Authors' contributions}

PW and SY conceived the research; PW and RG performed the experiments; PW and SY analyzed the data; PW, YY, and SY wrote the manuscript. All authors have read and approved the manuscript.

\section{Funding}

This work was supported by grants from the National Natural Science Foundation of China (31671656), the Fundamental Research Funds for the Central Universities (2662018YJ025), the National High Technology Research and Development Program of China (No. 2014AA10A600), and the National Research Foundation for the Doctoral Program of Higher Education of China (No. 20120146110013). The funding bodies had no role in the design of the study and collection, analysis, and interpretation of data and in writing the manuscript.

\section{Availability of data and materials}

All the supporting data are included within the article. The other dataset used and/or analyzed during the current study not included here are available from the corresponding author on request.

Ethics approval and consent to participate Not applicable.

Consent for publication Not applicable. 


\section{Competing interests}

The authors declare that they have no competing interests.

\section{Author details}

'National Key Laboratory of Crop Genetic Improvement, College of Plant Science and Technology, Huazhong Agricultural University, Wuhan, China. 2Present Address: Department of Agronomy and Horticulture, University of Nebraska Lincoln, Lincoln, NE, USA.

Received: 8 May 2019 Accepted: 24 September 2019

Published online: 01 November 2019

\section{References}

1. Izawa T. Adaptation of flowering-time by natural and artificial selection in Arabidopsis and rice. J Exp Bot. 2007;58(12):3091-7.

2. Gao H, Jin M, Zheng X, Chen J, Yuan D, Xin Y, Wang M, Huang D, Zhang Z, Zhou K, Sheng P, Ma J, Ma W, Deng H, Jiang L, Liu S, Wang H, Wu C, Yuan $\mathrm{L}$, Wan J. Days to heading 7, a major quantitative locus determining photoperiod sensitivity and regional adaptation in rice. Proc Natl Acad Sci. 2014;111(46):16337-42

3. Gouesnard B, Rebourg C, Welcker C, Charcosset A. Analysis of photoperiod sensitivity within a collection of tropical maize populations. Genet Resour Crop Ev. 2002:49(5):471-81

4. Turner A, Beales J, Faure S, Dunford RP, Laurie DA. The pseudo-response regulator $\mathrm{Ppd}-\mathrm{H} 1$ provides adaptation to photoperiod in barley. Science. 2005:310(5750):1031-4.

5. Murphy RL, Klein RR, Morishige DT, Brady JA, Rooney WL, Miller FR, Dugas DV, Klein PE, Mullet JE. Coincident light and clock regulation of pseudoresponse regulator protein 37 (PRR37) controls photoperiodic flowering in sorghum. Proc Natl Acad Sci. 2011;108(39):16469-74.

6. Goretti D, Martignago D, Landini M, Brambilla V, Gomez-Ariza J, Gnesutta N, Galbiati F, Collani S, Takagi H, Terauchi R, Mantovani R, Fornara F. Transcriptional and post-transcriptional mechanisms limit heading date 1 ( $\mathrm{Hd} 1$ ) function to adapt rice to high latitudes. PLoS Genet. 2017;13(1):e1006530.

7. Simpson GG, Dean C. Arabidopsis, the rosetta stone of flowering time? Science. 2002;296(5566):285-9.

8. Hayama R, Coupland $\mathrm{G}$. The molecular basis of diversity in the photoperiodic flowering responses of Arabidopsis and rice. Plant Physiol. 2004;135(2):677-84.

9. Searle I, Coupland G. Induction of flowering by seasonal changes in photoperiod. EMBO J. 2004;23(6):1217-22

10. Wu C, You C, Li C, Long T, Chen G, Byrne M, Zhang Q. RID1, encoding a Cys2/ His2-type zinc finger transcription factor, acts as a master switch from vegetative to floral development in rice. Proc Natl Acad Sci. 2008;105(35):12915-20.

11. Xue W, Xing Y, Weng X, Zhao Y, Tang W, Wang L, Zhou H, Yu S, Xu C, Li X Zhang Q. Natural variation in Ghd7 is an important regulator of heading date and yield potential in rice. Nat Genet. 2008:40(6):761-7.

12. Doi K, Izawa T, Fuse T, Yamanouchi U, Kubo T, Shimatani Z, Yano M, Yoshimura A. Ehd1, a B-type response regulator in rice, confers short-day promotion of flowering and controls FT-like gene expression independently of Hd1. Genes Dev. 2004;18(8):926-36.

13. Yamamoto $T$, Lin $H$, Sasaki $T$, Yano M. Identification of heading date quantitative trait locus Hd6 and characterization of its epistatic interactions with $\mathrm{Hd} 2$ in rice using advanced backcross progeny. Genetics. 2000;154(2):885-91.

14. Izawa T, Oikawa T, Sugiyama N, Tanisaka T, Yano M, Shimamoto K Phytochrome mediates the external light signal to repress FT orthologs in photoperiodic flowering of rice. Genes Dev. 2002;16(15):2006-20.

15. Ogiso E, Takahashi Y, Sasaki T, Yano M, Izawa T. The role of casein kinase II in flowering time regulation has diversified during evolution. Plant Physiol. 2010;152(2):808-20.

16. Matsubara K, Yamanouchi U, Nonoue $Y$, Sugimoto $K$, Wang ZX, Minobe $Y$, Yano M. Ehd3, encoding a plant homeodomain finger-containing protein, is a critical promoter of rice flowering. Plant J. 2011;66(4):603-12.

17. Shibaya T, Nonoue Y, Ono N, Yamanouchi U, Hori K, Yano M. Genetic interactions involved in the inhibition of heading by heading date QTL, Hd2 in rice under long-day conditions. Theor Appl Genet. 2011;123(7):1133-43.

18. Lin HX, Liang ZW, Sasaki T, Yano M. Fine mapping and characterization of quantitative trait loci $\mathrm{Hd} 4$ and $\mathrm{Hd} 5$ controlling heading date in rice. Breeding Sci. 2003;53:51-9.
19. Lin HX, Yamamoto T, Sasaki T, Yano M. Characterization and detection of epistatic interactions of 3 QTLs, Hd1, Hd2, and $\mathrm{Hd} 3$, controlling heading date in rice using nearly isogenic lines. Theor Appl Genet. 2000;101:1021-8.

20. Wang $P$, Xing YZ, Li ZK, Y U SB. Improving rice yield and quality by QTL pyramiding. Mol Breeding. 2012;29:903-13.

21. Wei X, Xu J, Guo H, Jiang L, Chen S, Yu C, Zhou Z, Hu P, Zhai H, Wan J. DTH8 suppresses flowering in rice, influencing plant height and yield potential simultaneously. Plant Physiol. 2010;153(4):1747-58.

22. Dai $X$, Ding $Y$, Tan L, Fu Y, Liu F, Zhu Z, Sun X, Sun X, Gu P, Cai H, Sun C. LHD1, an allele of DTH8/Ghd8, controls late heading date in common wild rice (Oryza rufipogon). J Integr Plant Biol. 2012;54(10):790-9.

23. Koo BH, Yoo SC, Park JW, Kwon CT, Lee BD, An G, Zhang Z, Li J, Li Z, Paek NC. Natural variation in OsPRR37 regulates heading date and contributes to rice cultivation at a wide range of latitudes. Mol Plant. 2013;6(6):1877-88.

24. Zhang ZY, Hu W, Shen GJ, Liu HY, Hu Y, Zhou XC, Liu TM, Xing YZ. Alternative functions of $\mathrm{Hd} 1$ in repressing or promoting heading are determined by Ghd7 status under long-day conditions. Sci Rep. 2017; 7(1):5388

25. Nemoto $Y$, Nonoue $Y$, Yano M, Izawa T. Hd1, a CONSTANS ortholog in rice, functions as an Ehd1 repressor through interaction with monocot-specific CCT-domain protein Ghd7. Plant J. 2016;86(3):221-33.

26. Zhang J, Zhou X, Yan W, Zhang Z, Lu L, Han Z, Zhao H, Liu H, Song P, Hu Y, Shen G, He Q, Guo S, Gao G, Wang G, Xing Y. Combinations of the Ghd7, Ghd8 and Hd1 genes largely define the ecogeographical adaptation and yield potential of cultivated rice. New Phytol. 2015;208(4):1056-66.

27. Yan W, Wang P, Chen H, Zhou H, Li Q, Wang C, Ding Z, Zhang Y, Yu S, Xing $Y$, Zhang Q. A major QTL, Ghd8, plays pleiotropic roles in regulating grain productivity, plant height, and heading date in rice. Mol Plant. 2011;4(2): 319-30

28. Yano M, Katayose $Y$, Ashikari M, Yamanouchi U, Monna L, Fuse T, Baba T, Yamamoto K, Umehara Y, Nagamura Y, Sasaki T. Hd1, a major photoperiod sensitivity quantitative trait locus in rice, is closely related to the Arabidopsis flowering time gene CONSTANS. Plant Cell. 2000;12(12):2473-84.

29. Hori K, Ogiso-Tanaka E, Matsubara K, Yamanouchi U, Ebana K, Yano M. $\mathrm{Hd16}$, a gene for casein kinase I, is involved in the control of rice flowering time by modulating the day-length response. Plant J. 2013;76(1):36-46.

30. Du A, Tian W, Wei M, Yan W, He H, Zhou D, Huang X, Li S, Ouyang X. The DTH8-Hd1 module mediates day-length-dependent regulation of rice flowering. Mol Plant. 2017;10(7):948-61.

31. Takahashi Y, Teshima KM, Yokoi S, Innan H, Shimamoto K. Variations in Hd1 proteins, Hd3a promoters, and Ehd1 expression levels contribute to diversity of flowering time in cultivated rice. Proc Natl Acad Sci. 2009;106(11):4555-60.

32. Zhu S, Wang J, Cai M, Zhang H, Wu F, Xu Y, Li C, Cheng Z, Zhang X, Guo X, Sheng P, Wu M, Wang J, Lei C, Wang J, Zhao Z, Wu C, Wang H, Wan J. The OSHAPL1-DTH8-Hd1 complex functions as the transcription regulator to repress heading date in rice. J Exp Bot. 2017;68(3):552-67.

33. Petroni K, Kumimoto RW, Gnesutta N, Calvenzani V, Fornari M, Tonelli C, Holt BF, Mantovani R. The promiscuous life of plant NUCLEAR FACTOR Y transcription factors. Plant Cell. 2012;24(12):4777-92.

34. Higo K, Ugawa Y, Iwamoto M, Korenaga T. Plant cis-acting regulatory DNA elements (PLACE) database. 1999. Nucleic Acids Res. 1999;27(1):297-300.

35. Gnesutta N, Kumimoto RW, Swain S, Chiara M, Siriwardana C, Horner DS, Holt BF, Mantovani R. CONSTANS imparts DNA sequence specificity to the histone fold NF-YB/NF-YC dimer. Plant Cell. 2017;29(6):1516-32.

36. Tiwari SB, Shen $Y$, Chang HC, Hou Y, Harris A, Ma SF, McPartland M, Hymus GJ, Adam L, Marion C, Belachew A, Repetti PP, Reuber TL, Ratcliffe OJ. The flowering time regulator CONSTANS is recruited to the FLOWERING LOCUS T promoter via a unique cis-element. New Phytol. 2010;187(1):57-66.

37. Zhao J, Huang X, Ouyang X, Chen W, Du A, Zhu L, Wang S, Deng XW, Li S. OsELF3-1, an ortholog of Arabidopsis early flowering 3, regulates rice circadian rhythm and photoperiodic flowering. PLoS One. 2012;7(8):e43705.

38. Yamamoto A, Kagaya Y, Toyoshima R, Kagaya M, Takeda S, Hattori T. Arabidopsis NF-YB subunits LEC1 and LEC1-LIKE activate transcription by interacting with seed-specific ABRE-binding factors. Plant J. 2009;58(5):843-56.

39. Xu JJ, Zhang XF, Xue HW. Rice aleurone layer specific OsNF-YB1 regulates grain filling and endosperm development by interacting with an ERF transcription factor. J Exp Bot. 2016;67(22):6399-411.

40. Bello BK, Hou Y, Zhao J, Jiao G, Wu Y, Li Z, Wang Y, Tong X, Wang W, Yuan W, Wei X, Zhang J. NF-YB1-YC12-bHLH144 complex directly activates Wx to regulate grain quality in rice (Oryza sativa L.). Plant Biotechnol J. 2019;17(7):1222-35. 
41. Hou XL, Zhou JN, Liu C, Liu L, Shen LS, Yu H. Nuclear factor Y-mediated H3K27me3 demethylation of the SOC1 locus orchestrates flowering responses of Arabidopsis. Nat Commun. 2014;5:4601.

42. Kumimoto RW, Zhang Y, Siefers N, Holt BF. NF-YC3, NF-YC4 and NF-YC9 are required for CONSTANS-mediated, photoperiod-dependent flowering in Arabidopsis thaliana. Plant J. 2010;63(3):379-91.

43. Bai AN, Lu XD, Li DQ, Liu JX, Liu CM. NF-YB1-regulated expression of sucrose transporters in aleurone facilitates sugar loading to rice endosperm. Cell Res. 2016;26(3):384-8.

44. Li CX, Distelfeld A, Comis A, Dubcovsky J. Wheat flowering repressor VRN2 and promoter $\mathrm{CO} 2$ compete for interactions with NUCLEAR FACTOR-Y complexes. Plant J. 2011;67(5):763-73.

45. Fujino K, Yamanouchi U, Yano M. Roles of the Hd5 gene controlling heading date for adaptation to the northern limits of rice cultivation. Theor Appl Genet. 2013;126(3):611-8.

46. Gomez-Ariza J, Galbiati F, Goretti D, Brambilla V, Shrestha R, Pappolla A, Courtois $B$, Fornara F. Loss of floral repressor function adapts rice to higher latitudes in Europe. J Exp Bot. 2015;66(7):2027-39.

47. Wang C, Chen S, Yu S. Functional markers developed from multiple loci in GS3 for fine marker-assisted selection of grain length in rice. Theor Appl Genet. 2011;122(5):905-13.

48. Wen W, Mei H, Feng F, Yu S, Huang Z, Wu J, Chen L, Xu X, Luo L. Population structure and association mapping on chromosome 7 using a diverse panel of Chinese germplasm of rice (Oryza sativa L.). Theor Appl Genet. 2009;119(3):459-70.

49. Livak KJ, Schmittgen TD. Analysis of relative gene expression data using real-time quantitative PCR and the 2(-Delta Delta C(T)) method. Methods. 2001;25(4):402-8.

50. Zhao Y, Cheng S, Song Y, Huang Y, Zhou S, Liu X, Zhou DX. The interaction between rice ERF3 and WOX11 promotes crown root development by regulating gene expression involved in cytokinin signaling. Plant Cell. 2015; 27(9):2469-83.

51. Zhao Y, Hu Y, Dai M, Huang L, Zhou DX. The WUSCHEL-related homeobox gene WOX11 is required to activate shoot-borne crown root development in rice. Plant Cell. 2009:21(3):736-48

52. Statistica. StatSoft Incorporated, Tusla, OK, USA. Accessed 21 Apr 2015

53. JMP, Version 12.0.1. SAS Institute Inc., Cary, NC. 1989-2017. Accessed 22 July 2017

\section{Publisher's Note}

Springer Nature remains neutral with regard to jurisdictional claims in published maps and institutional affiliations.

Ready to submit your research? Choose BMC and benefit from:

- fast, convenient online submission

- thorough peer review by experienced researchers in your field

- rapid publication on acceptance

- support for research data, including large and complex data types

- gold Open Access which fosters wider collaboration and increased citations

- maximum visibility for your research: over $100 \mathrm{M}$ website views per year

At $\mathrm{BMC}$, research is always in progress.

Learn more biomedcentral.com/submissions 